\title{
Sedation and analgesia in patients on mechanical ventilation in pediatric intensive care units in Argentina
}

\author{
Pedro Taffarel, M.D. ${ }^{a, b}$, German Bonetto, M.D. ${ }^{c}$, Facundo Jorro Barón, M.D. ${ }^{a}$ \\ and Claudia Meregalli, M.D. ${ }^{a}$
}

\begin{abstract}
Introduction. Children in pediatricintensive care units (PICUs) are exposed to experiencing pain, stress and anxiety due to their disease, treatment or care setting. Adequate sedation and analgesia are key to their care, particularly in patients requiring mechanical ventilation $(\mathrm{MV})$.

Objective. To determine the usual practice in sedation and analgesia management in patients requiring MV in PICUs in Argentina.

Material and methods. Descriptive, crosssectional, multi-center study conducted by means of e-mailed surveys.

Results. A total of 45 PICUs were surveyed, $18 \%$ $(\mathrm{N}=8)$ of which follow a sedation and analgesia protocol strictly, while $58 \%(\mathrm{~N}=26)$ follow an "implied" protocol based on routine practice. The most commonly used drugs were midazolam, for sedation, and fentanyl, for analgesia. In 31\% $(\mathrm{N}=14)$ of the PICUs, sedation was monitored through assessment scales (modified Ramsay and/or Comfort scales). In $4 \%(\mathrm{~N}=2)$ of units, daily, scheduled interruptions of sedation was implemented. In patients who are difficult to sedate, dexmedetomidine was the most commonly used adjuvant. In $73 \%(\mathrm{~N}=33)$ of the units, neuromuscular blocking agents were used in compliance with precise guidelines and under clinical monitoring. In $20 \%$ ( $\mathrm{N}=9$ ) of the PICUs there was a sedation and analgesia weaning protocol in place, and morphine and lorazepam are the most commonly used drugs.

Conclusion. Only a low percentage of surveyed PICUs had a protocol in place for the routine management of sedation and analgesia in patients on MV.

Key words: sedation, analgesia, mechanical ventilation.
\end{abstract}

http: / / dx.doi.org/10.5546/ aap.2018.eng.e196

To cite: Taffarel P, Bonetto G, Jorro Barón F, et al. Sedation and analgesia in patients on mechanical ventilation in pediatric intensive care units in Argentina. Arch Argent Pediatr 2018;116(2):e196-e203.
Funding:

None.

Conflict of interest:

None.

Received: 5-22-2017

Accepted: 9-6-2017

\section{INTRODUCTION}

Patients hospitalized in pediatric intensive care units (PICUs) are exposed to experiencing pain, stress and anxiety due to their disease, treatment or care setting. Adequate sedation and analgesia are key components in their care, particularly in patients requiring mechanical ventilation (MV), in whom the purpose of sedation is to reduce pain, anxiety and agitation, induce amnesia, facilitate the adjustment of the ventilator and avoid adverse events that may be likely to threaten patients' safety. ${ }^{1}$

The ideal situation is one that allows the patient to be calm, sensitive to stimuli and properly connected to the ventilator, and is associated with a reduced number of days on MV and hospital stay; ${ }^{2}$ this goal, however, is hard to achieve in pediatrics. Consensus guidelines developed by an expert panel recommend the use of monitored protocols for optimized control of sedation and analgesia. ${ }^{1,3-5}$ This paper is aimed at reporting the usual practice in sedation and analgesia management in patients requiring MV in Argentine PICUs.

\section{OBJECTIVE}

To determine the usual practice in sedation and analgesia management in patients requiring MV in PICUs in Argentina.

\section{MATERIALS AND METHODS Study design}

Descriptive, cross-sectional, multicenter study.

\section{Methodology}

The study was conducted by 
means of surveys and participants were selected by convenience sampling. Surveys were e-mailed to different PICUs of Argentina belonging to the public, private and social security settings. A questionnaire consisting of 10 multiplechoice questions was developed (Annex 1). The questionnaire was e-mailed to a representative (unit head, staff physician or medical coordinator) of each PICU invited to cooperate during March 2017. The following month, the completed surveys were collected and analyzed.

The frequency of use of a certain drug was classified as occasionally, frequently or always, depending on whether the drug was used in less than $50 \%, 50 \%$ to $80 \%$, or more than $80 \%$ of patients, respectively.

\section{Statistical analysis}

Data were expressed as medians and their respective interquartile ranges or as percentages, and analyzed using the STATA 13 (StataCorp, LT) software.

\section{RESULTS}

A total of 45 PICUs from 43 healthcare institutions distributed in different geographic areas of Argentina responded to the survey (see Table 1). Forty-nine percent $(21 / 43)$ of the facilities belong to the public healthcare system, while 33\% $(15 / 45)$ are pediatric or mother-and-child care facilities. Five PICUs provide specific care: 3 are for cardiovascular recovery, 1 is a burn care center and 1 is for immunosuppressed patients. The remaining 40 are multipurpose and 14 of them have a cardiovascular recovery unit. The median number of PICU beds is 8 (6-12).

TABLE 1. Geographic distribution of the pediatric intensive care units surveyed

\begin{tabular}{lc}
\hline City & $\begin{array}{c}\text { Number of } \\
\text { institutions }\end{array}$ \\
\hline Autonomous City of Buenos Aires & 15 \\
Córdoba & 9 \\
Province of Buenos Aires, Greater Buenos Aires & 9 \\
Province of Buenos Aires, outside of GBA & 2 \\
Tucumán & 2 \\
Chaco & 1 \\
Mendoza & 1 \\
Salta & 1 \\
Misiones & 1 \\
San Luis & 1 \\
San Juan & 1 \\
\hline
\end{tabular}

GBA: Greater Buenos Aires.
In $18 \%$ of the PICUs $(\mathrm{N}=8)$, a sedation and analgesia protocol is strictly followed, while $58 \%$ $(\mathrm{N}=26)$ follow an implied protocol based on the PICU staff's routine practice for sedation and analgesia management in patients on MV, and $24 \%(\mathrm{~N}=11)$ does not use a protocol and each member of the treating team conducts his or her own sedation and analgesia management.

Ninety-eight percent $(\mathrm{N}=44)$ of PICUs start by administering continuous IV sedation and analgesia; only one unit begins with intermittent sedation and continuous analgesia. Midazolam and ketamine are used in $100 \%$ of the PICUs, and the former is used more frequently. Propofol is used in $29 \%$ of the PICUs surveyed, although not frequently, and nearly $20 \%$ of the PICUs use thiopental. Fentanyl and morphine are used as opioid analgesics in 100\% of the PICUs, with the former being the most commonly administered. Ibuprofen is the most widely used nonopioid analgesic; in general, it is administered occasionally or frequently (see Table 2).

To monitor the sedation level, $31 \%(\mathrm{~N}=14)$ of the PICUs use some assessment scale: modified Ramsay and / or Comfort scales; 1 unit uses the bispectral index (BIS) and 69\% (N=31) use nurse or physician clinical assessment based on patients' physiological parameters, movements and well-being.

Only 2 units have a scheduled strategy in place for daily sedation interruption (DSI).

In patients who are difficult to sedate, PICUs use different drugs as adjuvants. Dexmedetomidine, chloral hydrate, clonidine are the most commonly used adjuvants, while propofol and phenobarbital are less frequently administered (see Table 3).

In $73 \%(\mathrm{~N}=33)$ of the units, neuromuscular blocking agents (NMBAs) are used for precise indications (severe traumatic brain injury, high MV parameters, decreased metabolic expenditure), while in the remaining 27\% $(\mathrm{N}=12)$ they are routinely administered from MV initiation until weaning. Monitoring of neuromuscular blockade level and its ensuing administration is performed by nurse and/ or physician assessment based on the patient's movement and well-being.

In $20 \%(\mathrm{~N}=9)$ of the PICUs there is a sedation and analgesia weaning protocol in place, while in $80 \%(\mathrm{~N}=36)$ weaning is tailored to each patient; morphine and lorazepam are the most commonly used drugs (see Figure 1). 
e198 / Arch Argent Pediatr 2018;116(2):e196-e203 / Original article

TABLE 2. Frequency of use of the different sedative and analgesic drugs in pediatric intensive care units

\begin{tabular}{|c|c|c|c|c|c|c|}
\hline & & Drug & $\begin{array}{l}N^{o} \text { of PICUs } \\
\text { using it (\%) }\end{array}$ & $\begin{array}{c}\text { Occasionally } \\
(<50 \% \text { of pts.) }\end{array}$ & $\begin{array}{c}\text { Frequently } \\
(50-80 \% \text { of pts.) }\end{array}$ & $\begin{array}{c}\text { Always } \\
\text { (> } 80 \% \text { of pts.) }\end{array}$ \\
\hline \multirow[t]{9}{*}{ Sedatives } & Anesthetics & Thiopental & $8(18 \%)$ & $8(100 \%)$ & - & - \\
\hline & & Etomidate & $0(0 \%)$ & - & - & - \\
\hline & & Propofol & $13(29 \%)$ & $13(100 \%)$ & - & - \\
\hline & & Ketamine & $45(100 \%)$ & $20(44 \%)$ & $21(47 \%)$ & $4(9 \%)$ \\
\hline & Anxiolytics- & Diazepam & $15(33 \%)$ & $13(87 \%)$ & $2(13 \%)$ & - \\
\hline & hypnotics & Midazolam & $45(100 \%)$ & - & $9(20 \%)$ & $36(80 \%)$ \\
\hline & & Chlorpromazine/ & & & & \\
\hline & & levomepromazine & $37(82 \%)$ & $28(76 \%)$ & $9(24 \%)$ & - \\
\hline & & Chloral hydrate & $40(89 \%)$ & $20(50 \%)$ & $15(38 \%)$ & $5(12 \%)$ \\
\hline \multirow[t]{8}{*}{ Analgesics } & Non- & Acetylsalicylic acid & $3(7 \%)$ & $3(100 \%)$ & - & - \\
\hline & opioids & Ibuprofen & $41(91 \%)$ & $16(39 \%)$ & $21(51 \%)$ & $4(10 \%)$ \\
\hline & & Paracetamol & $34(75 \%)$ & $19(56 \%)$ & $12(35 \%)$ & $3(9 \%)$ \\
\hline & Opioids & Morphine & $45(100 \%)$ & $2(4 \%)$ & $35(80 \%)$ & $8(16 \%)$ \\
\hline & & Fentanyl & $45(100 \%)$ & - & $11(24 \%)$ & $34(76 \%)$ \\
\hline & & Remifentanil & $13(29 \%)$ & $13(100 \%)$ & - & - \\
\hline & & Meperidine & $5(11 \%)$ & $5(100 \%)$ & - & - \\
\hline & & Codeine & $9(20 \%)$ & $9(100 \%)$ & - & - \\
\hline
\end{tabular}

No.: Number; PICU: Pediatric Intensive Care Unit; pts.: patients.

TABLE 3. Frequency of use of the different adjuvant sedatives in patients who are difficult to sedate

\begin{tabular}{|c|c|c|c|c|}
\hline Drug & $\begin{array}{c}\text { No. of PICUs } \\
\text { using it }(\%)\end{array}$ & $\begin{array}{l}\text { Occasionally } \\
\text { (<50\% of pts.) }\end{array}$ & $\begin{array}{c}\text { Frequently } \\
(50-80 \% \text { of pts.) }\end{array}$ & $\begin{array}{c}\text { Always } \\
\text { (> 80\% of pts.) }\end{array}$ \\
\hline Clonidine & $36(80 \%)$ & $15(42 \%)$ & $11(30 \%)$ & $10(28 \%)$ \\
\hline Dexmedetomidine & $37(82 \%)$ & $3(8 \%)$ & $17(46 \%)$ & $17(46 \%)$ \\
\hline Propofol & $7(16 \%)$ & $7(100 \%)$ & - & - \\
\hline Ketamine & $39(87 \%)$ & $19(49 \%)$ & $18(46 \%)$ & $2-5 \%$ \\
\hline Chlorpromazine/levomepromazine/ & $36(80 \%)$ & $23(64 \%)$ & $10(28 \%)$ & $3(8 \%)$ \\
\hline Chloral hydrate & $37(82 \%)$ & $12(32 \%)$ & $15(41 \%)$ & $10(27 \%)$ \\
\hline Phenobarbital & $9(20 \%)$ & $9(100 \%)$ & - & - \\
\hline Thiopental & $2(4.5 \%)$ & $2(100 \%)$ & - & - \\
\hline
\end{tabular}

No.: Number; PICU: Pediatric Intensive Care Unit; pts.: patients.

FIGURE 1. Frequency of use of different drugs for treating sedation and analgesia weaning abstinence symptoms in pediatric intensive care units

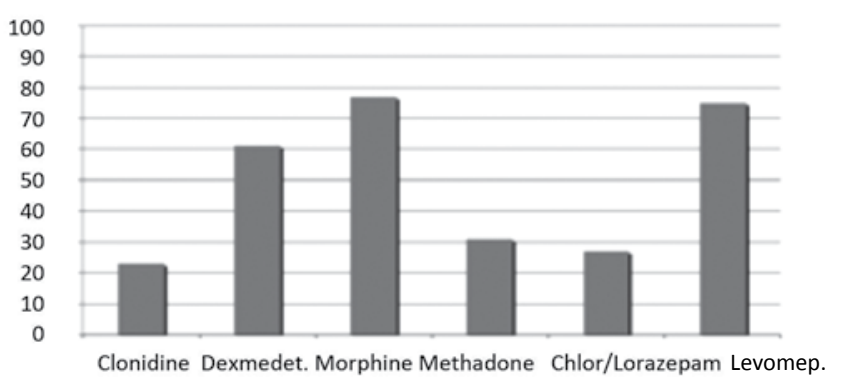

Sedation and analgesia weaning drugs

Dexmedet.: dexmedetomidine; Chlor.: chlorpromazine; Levomep.: levomepromazine. 


\section{DISCUSSION}

The use of sedation and analgesia protocols is associated with good outcomes in critically ill adult patients. This association has not been evidenced in pediatrics. A study comparing the use of a sedation protocol (17 PICUs, $n=1225$ ) against usual management (14 PICUs, $\mathrm{n}=1224$ ) in pediatric patients on MV for acute respiratory failure did not report differences in the number of days on MV: 6.5 days (IQR: 4.1-11.2) in the protocolized group and 6.5 days (IQR: 3.7$12.1)$ in the control group $(p=0.61)$, nor in the development of sedation-related adverse events. ${ }^{8}$

The most commonly used drugs are midazolam for sedation, and fentanyl for analgesia, and their combination is the most frequent in Argentine PICUs. These data are consistent with what has been reported in the bibliography. ${ }^{7-9}$

Traditionally, the use of propofol for maintenance of long-term sedation in critically ill children has been discouraged and is not licensed due to the risk of propofol infusion syndrome (PIS), characterized by metabolic acidosis, heart failure and, at least, one of the following signs: rhabdomyolysis, hypertriglyceridemia and renal failure. ${ }^{10,11}$ Until 2008, 33 pediatric cases were reported, ${ }^{12}$ with a survival rate of $36 \%$. In $29 \%$ of the PICUs in our study propofol is occasionally used. This data is consistent with the increasingly more frequent and safer use reported in the literature. ${ }^{11,13}$ In a survey conducted in Germany, ${ }^{14}$ $79 \%$ of units surveyed (145 out of 184) used it, and the main indications were difficult sedation (44\%), postoperative ventilation $(43 \%)$ and difficult extubation (30\%); 7 cases of PIS were reported.

DSI has been shown to reduce the number of days on MV and the length of hospital stay in the adult population. ${ }^{15}$ In this study, only $4 \%$ of the units used this strategy. In three studies conducted in pediatric patients, contradictory results were observed. While Gupta et al. and Verlaat et al., in single-center studies, with 102 and 30 patients, respectively, reported fewer days on MV and shorter hospital stays for the DSI group, the Vet et al. study, conducted in three units with 129 patients, did not evidence differences in days off MV or length of hospital stay in protocolized sedation (PS) groups and the DSI + PS group. Mortality was higher in the DSI + PS group (6/66) than in the PS group $(0 / 63)$ $(\mathrm{p}=0.03){ }^{16-18}$

The ideal sedation is the one that allows the patient to be calm, sensitive to stimuli and comfortable with the MV, and is associated with a reduction in the number of days on MV and in the length of stay in intensive care unit, without an increase in unscheduled extubations. ${ }^{2}$ In order to accomplish this goal, assessing the sedation level becomes essential. The frequency of use of sedation scales is low in pediatrics. Kudchadkar ${ }^{19}$ and Mencía ${ }^{9}$ reported that pediatric patients were assessed by means of sedation scales in $42 \%$ and $45 \%$ of the cases, respectively. Ramsay scale was the most widely used, with BIS being used in 50\% of PICUs.

In relation to difficult sedation, in our study, dexmedetomidine was the most commonly used adjuvant. Grant ${ }^{20}$ assessed the use of dexmedetomidine in patients on MV due to respiratory failure; $49 \%$ of patients from 31 PICUs received this drug. Patients were classified in three groups depending on whether they were receiving dexmedetomidine as primary sedative $(\mathrm{n}=138 ; 11 \%)$, secondary sedative $(\mathrm{n}=280 ; 23 \%)$ or periextubation agent $(n=178 ; 15 \%)$. They concluded that the use of dexmedetomidine as primary sedative in patients who had obtained the lowest severity score (< Pediatric Risk of Mortality III) attained adequate sedation levels rapidly. The use of dexmedetomidine as secondary agent did not appear to add benefits. Meanwhile, in the periextubation group, the use of dexmedetomidine facilitated MV weaning and reduced the number of days.

The role of dexmedetomidine as adjuvant agent in patients requiring long-term sedation and analgesia was also studied. Whalen ${ }^{21}$ conducted a retrospective study to determine the impact of its use on the required opioid and benzodiazepine dose in a cohort of 98 criticallyill subjects (neonate and pediatric patients), and concluded that there was no decrease in the opioid and benzodiazepine doses. In another study, Tobias et al. ${ }^{22}$ compared three groups, each composed of 10 pediatric patients on MV. Two of the groups were sedated with dexmedetomidine at different doses $(0.25$ and $0.5 \mathrm{mcg} / \mathrm{kg} / \mathrm{h})$ and the third group, with midazolam $(0.1 \mathrm{mg} /$ $\mathrm{kg} / \mathrm{h}$ ). All three groups received analgesia with intermittent morphine administration. Sedation as assessed by the Ramsay scale and BIS was equivalent in the 3 groups. A decrease in the requirements for morphine was found in the $0.5 \mathrm{mcg} / \mathrm{kg} / \mathrm{h}$ dexmedetomidine group versus the midazolam group. There is little evidence to support the use of chloral hydrate in critically-ill pediatric patients. Parkinson et al., 
in a controlled trial with 44 patients, evaluated sedation efficacy by comparing a combination of chloral hydrate and promethazine to a continuous intravenous midazolam infusion, which yielded better outcomes to the first group..$^{23}$ There is also little evidence in the literature regarding the use of levomepromazine in patients who are difficult to sedate: only case reports are available. ${ }^{24}$

No PICU reported the need for neuromuscular blockade. The most commonly used method to monitor the depth of neuromuscular blockade is peripheral nerve stimulation with train-of-four monitoring. However, as opposed to adult care units ${ }^{25}$ it is hardly used in PICUs.

As regards MV weaning, only $20 \%(\mathrm{~N}=9)$ of PICUs surveyed followed a sedation and analgesia weaning protocol strictly. The abstinence syndrome (AS) had a 34-70\% prevalence on patients with sedation and analgesia. ${ }^{26}$ It was associated with increased morbidity, length of hospital stay and psychological disorders. The use of methadone and/or dexmedetomidine to facilitate weaning from continuous opioid infusion played a leading role in our study. A recent meta-analysis that included twelve studies involving 459 pediatric patients concluded that there was no sufficient evidence to recommend any particular methadone weaning strategy, or to recommend methadone over other medications. ${ }^{27}$ Oschman et al. analyzed four publications of limited design and a total of 20 pediatric patients, and suggested that dexmedetomidine could potentially be beneficial for preventing and/or treating AS. ${ }^{28}$

This study reflects the use of sedatives and analgesics in some PICUs in Argentina. Its limitations include the use of self-report surveys, sampling by convenience and only one sampletaking per PICU. However, $100 \%$ of recipients answered and completed the survey in full. The importance of this paper lies in the fact that it gives insight into the situation of sedative and analgesic use across the country, and allows us to compare it with the bibliography to perform a diagnosis based on which we will be able to improve our treatment practices.

\section{CONCLUSION}

In Argentina, there is a low level (18\%) of protocolized practice in the management of sedation and analgesia in patients on $\mathrm{MV}$, as well as a certain degree of heterogeneity in its use. The most commonly used drugs were midazolam, for sedation, and fentanyl, for analgesia, and their combination is the most frequent, consistent with what has been reported in the literature. Dexmedetomidine had a leading role as adjuvant in the case of patients who are difficult to sedate and in the suppression of narcotics and benzodiazepines.

\section{Acknowledgments}

To the heads, coordinators and assistants of the pediatric intensive care units and their institutions that collaborated with their responses in this survey.

\section{REFERENCES}

1. Keogh S, Long D, Horn D. Practice guidelines for sedation and analgesia management of critically ill children: a pilot study evaluating guideline impact and feasibility in the PICU. BMJ Open 2015;5(3):e006428.

2. Hughes C, Girard T, Pandharipande P. Daily sedation interruption versus targeted light sedation strategies in ICU patients. Crit Care Med 2013;41(9):S39-45.

3. Playfor S, Thomas D, Choonara I. Sedation and neuromuscular blockade in paediatric intensive care: a review of current practice in the UK. Paediatr Anaesth 2003;13(2):147-51.

4. Playfor S, Jenkins I, Boyles C, et al. Consensus guidelines on sedation and analgesia in critically ill children. Intensive Care Med 2006;32(8):1125-36.

5. Tobias J. Sedation and analgesia in the pediatric intensive care unit. Pediatr Ann 2005;34(8):636-45.

6. Curley MAQ, Wypij D, et al. Protocolized sedation vs usual care in pediatric patients mechanically ventilated for acute respiratory failure: a randomized clinical trial. JAMA 2015;313(4):379-89.

7. Twite MD, Rashid A, Zuk J, et al. Sedation, analgesia, and neuromuscular blockade in the pediatric intensive care unit: survey of fellowship training programs. Pediatr Crit Care Med 2004;5(6):521-32.

8. Nolent P, Laudenbach V. Sédation et analgésie en réanimation - Aspects pédiatriques. Ann Fr Anesth Reanim 2008;27(7-8):623-32.

9. Mencía S, Botrán M, López-Herce J, et al. Manejo de la sedation and analgesia y de los relajantes musculares en las unidades de cuidados intensivos pediátricos españolas. An Pediatr (Barc). 2011;74(6):396-404.

10. Bray RJ. The propofol infusion syndrome in infants and children: can we predict the risk. Curr Opin Anaesthesiol 2002;15(3):339-42.

11. Agudelo S, Mencía S, Faro A, et al. Propofol en perfusión continúa en niños en estado crítico. Med Intensiva 2012;36(6):410-5.

12. Corbett S, Montoya I, Moore F. Propofol-related infusion syndrome in intensive care patients. Pharmacotherapy 2008;28(2):250-8.

13. Koriyama H, Duff J, Guerra G, et al. Is propofol a friend or a foe of the pediatric intensivist? Description of propofol use in a PICU. Pediatr Crit Care Med 2014;15(2):e66-71.

14. Kruessell M, Udink ten Cate F, Kraus A, et al. Use of propofol in pediatric intensive care units: a national survey in Germany. Pediatr Crit Care Med 2012;13(3):e150-4.

15. Kress J, Pohlman A, O'Connor M, et al. Daily interruption of sedative infusions in critically ill patients undergoing mechanical ventilation. N Engl J Med 2000;342(20):1471-7.

16. Gupta K, Gupta V, Jayashree M, et al. Randomized 
controlled trial of interrupted versus continuous sedative infusions in ventilated children. Pediatr Crit Care Med 2012;13(2):131-5.

17. Verlaat C, Heesen G, Vet N, et al. Randomized controlled trial of daily interruption of sedatives in critically ill children. Paediatr Anaesth 2014;24(2):151-6.

18. Vet N, DeWildtS, VerlaatC, et al. A randomized controlled trial of daily sedation interruption in critically ill children. Intensive Care Med 2016;42(2):233-44.

19. Kudchadkar S, Yaster M, Punjabi N. Sedation, sleep promotion, and delirium screening practices in the care of mechanically ventilated children: a wake-up call for the pediatric critical care community. Crit Care Med 2014;42(7):1592-600.

20. Grant M, Schneider J, Asaro L, et al. Dexmedetomidine Use in Critically Ill Children With Acute Respiratory Failure. Pediatr Crit Care Med 2016;17(12):1131-41.

21. Whalen L, Di Gennaro J, Irby G, et al. Long-Term Dexmedetomidine Use and Safety Profile Among Critically Ill Children and Neonates. Pediatr Crit Care Med 2014;15(8):706-14.

22. Tobias J, Berkenbosch J. Sedation during mechanical ventilation in infants and children: Dexmedetomidine versus midazolam. South Med J 2004;97(5):451-5.

23. Parkinson L, Hughes J, Gill A, et al. A randomized controlled trial of sedation in the critically ill. Paediatr Anaesth 1997;7(5):405-10.

24. Van derZwaanS, Blankespoor R, Wolters A, etal. Additional use of methotrimeprazine for treating refractory agitation in pediatric patients. Intensive Care Med 2012;38(1):175-6.

25. Murray M, DeBlock H, Erstad B, et al. Clinical Practice Guidelines for Sustained Neuromuscular Blockade in the Adult Critically Ill Patient. Crit Care Med 2016;44(11):20792103.

26. Motta E, Luglio M, Delgado A, et al. Importance of the use of protocols for the management of analgesia and sedation in pediatric intensive care unit. Revista Assoc Med Bras (1992) 2016;62(6):602-9.

27. Dervan L, Yaghmai B, Watson RS, et al. The use of methadone to facilitate opioid weaning in pediatric critical care patients: a systematic review of the literature and metaanalysis. Paediatr Anaesth 2017;27(3):228-39.

28. Oschman A, McCabe T, Kuhn RJ. Dexmedetomidine for opioid and benzodiazepine withdrawal in pediatric patients. Am J Health Behav 2011;68(13):1233-8. 


\section{ANNEX}

\section{Survey about sedation and analgesia in patients on mechanical ventilation in pediatric intensive care units in Argentina}

Respondent's Name:

Institution:

Type of PICU: - Multipurpose:

- Specific: Type:

Number of Beds:

1. With regard to the existence of a sedation and analgesia PROTOCOL in your institution, in your opinion:

a) Yes, there is a written protocol in place and it is strictly followed.

b) There is no written protocol, but PICU staff's routine practice for the management of sedation and analgesia in patients on mechanical ventilation converges into an "implied protocol".

c) There is no protocol and each treating practitioner performs his or her own sedation and analgesia management.

2. When sedation and analgesia is initiated on a patient that is connected to MV, what is the most common combination and administration modality in your institution?
a) Continuous sedation and analgesia.
b) Intermittent sedation and analgesia.
c) Continuous sedation and intermittent analgesia.
d) Continuous analgesia and intermittent sedation.

3. What continuous sedation and analgesia is most frequently used in your institution?
a) Fentanyl and midazolam.
b) Morphine and midazolam.
c) Dexmedetomidine.
d) Others (Specify).

4. How often are the following sedative and analgesic drugs used in your institution? (Mark with an X).

\begin{tabular}{|c|c|c|c|c|c|c|}
\hline & & Drugs & $\begin{array}{c}\text { Never } \\
(0 \% \text { of pts. })\end{array}$ & $\begin{array}{c}\text { Occasionally } \\
(<50 \% \text { of pts. })\end{array}$ & $\begin{array}{c}\text { Frequently } \\
(50-80 \% \text { of pts.) }\end{array}$ & $\begin{array}{c}\text { Always } \\
(>80 \% \text { of pts.) }\end{array}$ \\
\hline \multirow[t]{2}{*}{ Sedatives } & Anesthetics & $\begin{array}{l}\text { Thiopental } \\
\text { Etomidate } \\
\text { Propofol } \\
\text { Ketamine }\end{array}$ & & & & \\
\hline & Anxiolytics-hypnotics & $\begin{array}{l}\text { Diazepam } \\
\text { Midazolam } \\
\text { Chlorpromazine/ } \\
\text { levomepromazine } \\
\text { Chloral hydrate }\end{array}$ & & & & \\
\hline \multirow[t]{2}{*}{ Analgesics } & Non-opioids & $\begin{array}{l}\text { Acetylsalicylic acid } \\
\text { Ibuprofen } \\
\text { Paracetamol }\end{array}$ & & & & \\
\hline & Opioids & $\begin{array}{l}\text { Morphine } \\
\text { Fentanyl } \\
\text { Remifentanil } \\
\text { Meperidine } \\
\text { Codeine }\end{array}$ & & & & \\
\hline
\end{tabular}


5. In relation to sedation and analgesia monitoring, your institution uses the following method:

a) Assessment scale (modified Ramsay and/or COMFORT).

b) Bispectral index (BIS).

c) Both methods mentioned in the items above.

d) Assessment of the patient's physiological parameters, movements and well-being, assessed by a nurse or an intensive care physician without using any given scale.

6. In relation to daily sedation interruption, your institution:

a) Uses it on a routine, protocolized basis.

b) Uses it sporadically on a non-protocolized basis.

c) Does not use it.

7. In the event that a patient is refractory to sedation with opioids and benzodiazepines, what adjuvants does your institution use? (Mark with an X).

\begin{tabular}{ccccc} 
Drugs & $\begin{array}{c}\text { Never } \\
(\mathbf{0} \% \text { of pts. })\end{array}$ & $\begin{array}{c}\text { Occasionally } \\
(<50 \% \text { of pts. })\end{array}$ & $\begin{array}{c}\text { Frequently } \\
(\mathbf{5 0 - 8 0} \% \text { of pts. })\end{array}$ & $\begin{array}{c}\text { Always } \\
(>80 \% \text { of pts. })\end{array}$ \\
\hline
\end{tabular}

Clonidine

Dexmedetomidine

Propofol

Ketamine

Chlorpromazine/Levomepromazine

Chloral hydrate

Phenobarbital

Thiopental

8. With respect to the use of neuromuscular blocking agents:

a) They are used on a routine basis from MV initiation until weaning.

b) They are used in particular situations (severe traumatic brain injury, high MV parameters, etc.).

9. With respect to monitoring of neuromuscular blocking agents:

a) Their administration is monitored and subject to nursing staff/intensive care physicians decision based on the patient's movements and well-being.

b) Their administration is monitored and subject to the muscle group's qualitative or quantitative response to peripheral nerve stimulation.

10. In relation to sedation and analgesia weaning, in your institution:

a) A written protocol is strictly followed.

b) There is no protocol in place and it is decided on a patient basis.

In relation to sedation and analgesia weaning, what are the most commonly used drugs in your institution to prevent the abstinence syndrome? (Mark with an X).

\begin{tabular}{llll} 
Methadone & Morphine & Chlorpromazine and / or levomepromazine & Diazepam \\
\hline Clonidine & Dexmedetomidine & Lorazepam & Other: \\
\hline
\end{tabular}

